\title{
HTLV-1-associated infective dermatitis and probable HTLV-1- associated myelopathy in an adolescent female*
}

\author{
Raquel Bisacotti Steglich ${ }^{1}$ \\ Paulo Ricardo Martins Souza ${ }^{2,3}$ \\ Rudimar dos Santos Riesgo ${ }^{4}$
}

\author{
Renata Elise Tonoli ${ }^{2}$ \\ Giselle Martins Pinto ${ }^{2}$
}

\begin{abstract}
A bstract: Human T cell lymphotropic virus type 1 (HTLV-1)-associated infective dermatitis (ID) is a chronic, severe and recurrent eczema occurring during childhood in patients vertically infected with HTLV-1. HTLV-1-associated myelopathy/tropical spastic paraparesia (HAM/ TSP) is slow and progressive. We report the case of an adolescent female from a non-endemic area for HTLV-1 who presents ID and, most likely, associated HAM/TSP. Keywords: Dermatitis; Demyelinating diseases; Human T-lymphotropic virus 1; Paraparesis, tropical spastic
\end{abstract}

\section{INTRODUCTION}

The HTLV-1 is a retrovirus of the family Retroviridae, genus Deltaretrovirus, which integrates into T-helper (CD4+) lymphocytes, leading to a lifelong cell infection. It is estimated that 10 to 20 million individuals are infected with HTLV-1 worldwide. Regions of HTLV-1 endemicity, with proportionately higher rates of infection, are clustered in Japan, the Caribbean, South and Central America, equatorial Africa, and Iran. No population study to date has reported on the real prevalence of HTLV-1 carriers in Brazil. However, Brazil is considered to have the largest absolute number of individuals infected with HTLV: about 2.5 million carriers. Salvador is the area with the highest prevalence rate $(1.76 \%){ }^{1-10}$

HTLV-1 is transmitted primarily through sexual contact. The second major mode of transmission is vertical transmission, followed by blood transfusions and use of contaminated material. In the case of intravenous transmissions, seroconversion occurs in $40-60 \%$ of recipients. Breastfeeding has a more import- ant role than intrauterine or perinatal transmission. There is a positive correlation between the duration of breastfeeding and proviral load in breast milk. ${ }^{2-9}$

\section{CASE REPORT}

16-year-old female patient, student, hospitalized, born and living in Porto Alegre/RS. Unknown perinatal history. The patient has had erythematous, scaly rash on the cephalic segment, trunk and large folds since early childhood. In adolescence, she developed erythema and yellowish, adherent and fetid crusts on the scalp, eyelid, ear and retroauricular eczema, and micropapular rash on the trunk (Figures 1, 2 and 3). She had painless cervical and axillary lymphadenopathy.

3 years earlier, she presented with progressive gait alteration and one year earlier, with urinary urge incontinence and constipation. Neurological examination revealed pyramidal syndrome, in which the lower limbs exhibit distal atrophy, spasticity, reduction in

Received on 18.02.2014

Approved by the Advisory Board and accepted for publication on 16.06.2014

Study conducted at the Santa Casa de Misericórdia de Porto Alegre - Porto Alegre (RS), Brazil.

Financial Support: None.

Conflict of Interest: None.

Universidade da Região de Joinville (UNIVILLE) - Joinville (SC), Brazil.

Santa Casa de Misericórdia de Porto Alegre - Porto Alegre (RS), Brazil.

Pontifícia Universidade Católica do Rio Grande do Sul (PUCRS) - Porto Alegre (RS), Brazil.

Universidade Federal do Rio Grande do Sul (UFRGS) - Porto Alegre (RS), Brazil.

(C2015 by Anais Brasileiros de Dermatologia 
force, hyperreflexia, Babinsky sign, bilateral clonus and parapareto-spastic gait.

Positive serology for HTLV-1 was detected by Western blot. Histopathology of the scalp revealed: moderate psoriasiform acanthosis, parakeratosis and mild spongiosis. Lymphocytic infiltrate with a predominance of CD8 + lymphocytes in the dermis (Figure 4). The urodynamics findings are compatible with neurogenic bladder. MRI showed atrophy of the thoracic spinal cord.

The patient showed cutaneous improvement when treated with sulfamethoxazole-trimethoprim and rapid recurrence of symptoms with discontinuation of treatment.

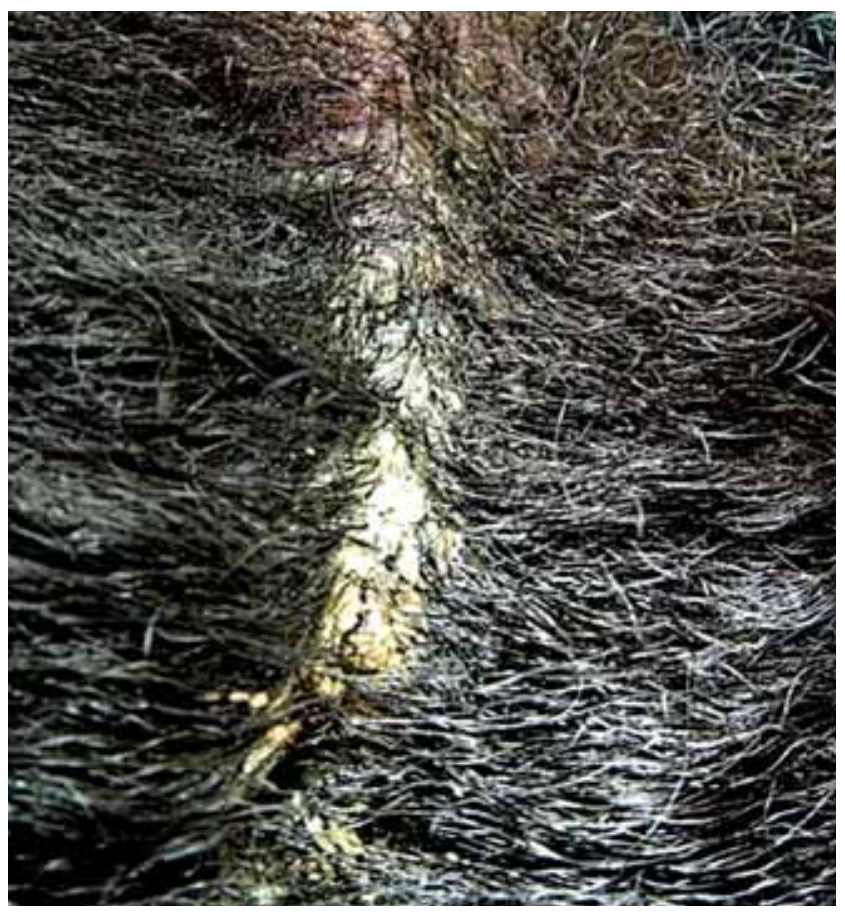

Figure 1: Yellowish, adherent crusts on the scalp

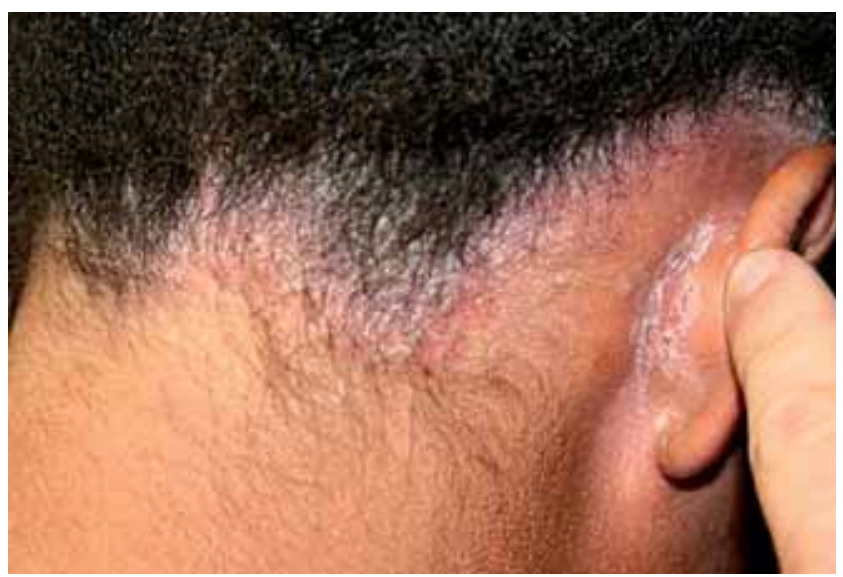

Figure 2: Retroauricular eczema and erythematous scaly rash on the scalp

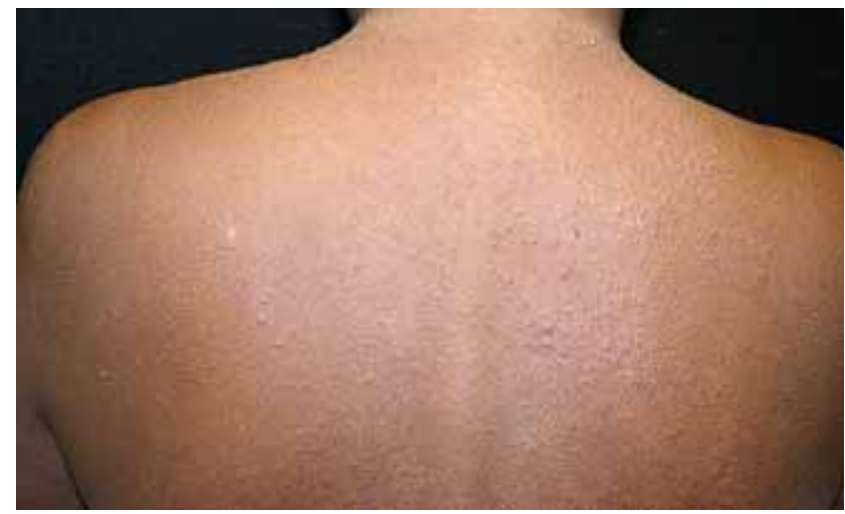

FIGURE 3: Micropapular rash on the trunk
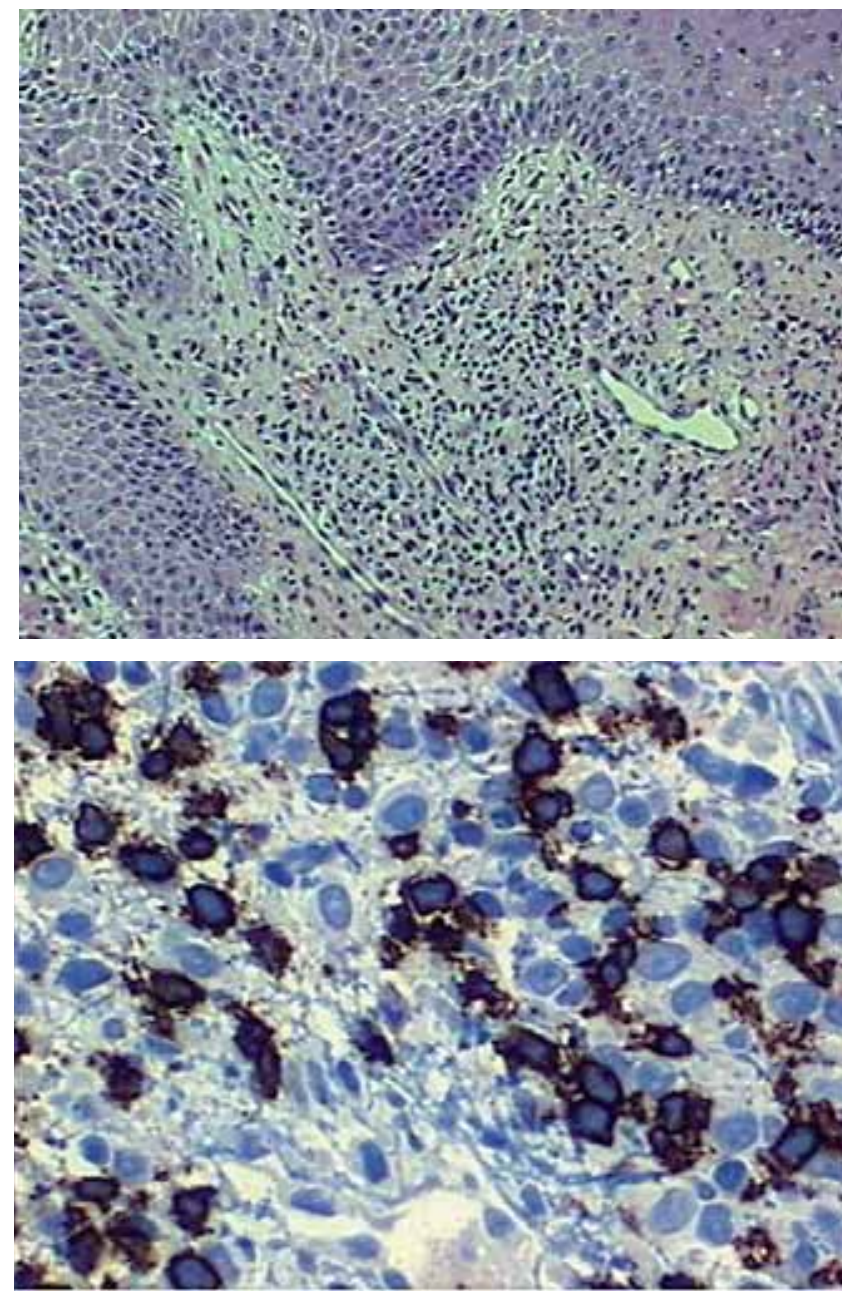

Figure 4: Histopathology of the scalp: moderate psoriasiform acanthosis, parakeratosis and mild spongiosis. Immunohistochemistry: lymphocytic infiltrate with a predominance of CD8 + lymphocytes in the dermis

\section{DISAUSSION}

Over $90 \%$ of individuals infected with HTLV-1 remain asymptomatic. Of these infected individuals, $3 \%$ develop adult $\mathrm{T}$ cell leukemia/lymphoma after 
a latency period of 20 years. Another 3\% develop HTLV-I-associated myelopathy/tropical spastic paraparesis (HAM/TSP), which is a severe and disabling disease, characterized by a slow and progressive demyelination of the pyramidal tracts. ${ }^{1,3}$ Its pathogenesis may involve an autoimmune reaction triggered by viruses or destructive cytotoxic effect of infected $\mathrm{T}$ cells. Gait disturbances, weakness, stiffness of the lower limbs and compromised dynamic balance are the main presenting symptoms of myelopathy. In 1986, new diagnostic criteria for HAM / TSP were established (Chart 1). 1,5,7-10

Some vertically infected children may develop infective dermatitis (ID). The onset of symptoms usually starts at 18 months of age and resolves during adolescence. The first cases with onset in adulthood have been recently described. ${ }^{2,4,7,8}$

At the time of the study by Lee et al, 298 cases of IDH had been confirmed. Most cases occur in Jamaica and Brazil. In the latter, the area with the highest incidence is Salvador/BA. One study showed that, in Jamaica, children vertically infected by HTLV-1 have a $2 \%$ chance of developing ID at the age of four. ${ }^{1,2,4}$

ID is characterized by a severe, recurrent, infected eczematous and exudative dermatitis with scaling and crusting primarily affecting the scalp, forehead, paranasal area, neck, retroauricular areas, armpits, and groins. ${ }^{2,5,10}$ Chronic watery rhinorrhea and crusts in the anterior nasal cavity are frequent characteristic findings, but are not always present. ${ }^{10}$ Other possible findings are blepharoconjunctivitis, generalized micropapular rash and lymphadenopathy . Recalcitrant bacterial skin infection by saprophytic organisms (Staphylococcus aureus and/or Streptococcus $\beta$-hae-

\section{Chart 1: Diagnostic Criteria of Osame et al HAM/ TSP (1986)}

1. Definitive HTLV-1 Associated Myelopathy (HAM)

1.1. Slowly progressive paraparesis, caused by symmetric myelopathy, predominantly involving pyramidal pathways.

1.2. Anti-HTLV-1

1.3. Anti-HTLV-1 antibodies present in liquor

2. Probable HAM

2.1 Paraparesis similar to the described in item 1.1 in patient with anti-HTLV-1 antibodies present in serum or liquor (but not in both) or

$2.2 \mathrm{c}$ inconsistent with the definition of item 1.1 in patient with anti-HTLV-1 antibodies present in serum and in liquor simultaneously

Source: Bittencourt AL, 2001' e Santos FLN, 2005. ${ }^{5}$ molyticus) also occurs. It is supposed that these symptoms would result from immunosuppressive effects of HTLV-1. ${ }^{1,2,4,10}$ In 1998, La Grenade et al., set diagnostic criteria for ID (Chart 2).

The recommended treatment ID is long-term systemic antibiotic-therapy until puberty, when the severity of the bacterial infection is expected to subside. Oral trimethoprim-sulfamethoxazole is the treatment of choice for its effectiveness, low cost and availability. ${ }^{5}$

Histology shows changes observed in other eczemas. It shows hyperkeratosis and/or parakeratosis, with crusts and acanthosis of varying degrees. We observe a mild infiltration of $\mathrm{T}$ lymphocytes in the dermis. Immunohistochemistry reveals the predominance of CD8 + cells in the skin and small percentage of cells with cytotoxic granules, suggesting that most CD8 + lymphocytes are not activated. ${ }^{1,2}$ Diagnosis depends on the confirmation of serum-reactivity by Western blot analysis or polymerase chain reaction (PCR). ${ }^{5}$

Epidemiological data suggest that ID in children can be a precursor to the development of ATLL or HAM/TSP in adolescence and adulthood. ${ }^{1,2,4,10}$ Primo et al, identified a $30 \%$ occurrence of HAM/TSP among patients previously diagnosed with ID. To date, only 6 cases of HAM/TSP associated with ID have been described, indicating a greater association between these two conditions than previously thought. The associa-

Chart 2: Diagnostic criteria of infectious dermatitis

\section{MAJOR CRITERIA}

1. Eczema in two or more areas: scalp, armpits, groin, outer ear, retroauricular region, eyelid margins, perinasal area and/or neck.

2. Chronic rhinorrhea without other signs of rhinitis and/ or crusted lesions in nasal vestibule

3. Chronic recurrent dermatitis with immediate response to antibiotic therapy but with relapse after cessation of treatment

4. Onset in childhood

5. Seropositivity for HTLV-1

\section{MINOR CRITERIA}

1. Positive cultures for Staphylococcus aureus and/or streptococcus $\beta$ - hemolytic in skin or nostrils

2. Papulous rash

3. Generalized lymphadenopathy with dermatopathic lymphadenitis

4. Anemia

5. Increased erythrocyte sedimentation rate

6. Hypergammaglobulinemia $\operatorname{IgD}$ and $\operatorname{IgE}$

7. Increased CD4 and CD8 and CD4/CD8 ratio

* The presence of items 1, 2 and 5 among major criteria, and at least two areas of the first item are mandatory to meet criterion 1. 
tion between ID and HAM/TSP in childhood and adolescence has been reported in less than 10 cases in the literature. ${ }^{3,4,5}$

The overactive immune response seen in ID and HAM/TSP indicates that both diseases may have a similar pathogenesis. The rapid progression of ID to HAM/TSP may be the result of a vigorous humoral anti-HTLV-1 response. The increased levels of antibodies seen in ID, together with the already defined role of humoral immunity in the development HAM/ TSP supports this theory.
ID may be the initial manifestation of HTLV-1 infection. Therefore, it can serve as an early clinical marker for HTLV-1 infection and an indicator of increased risk for the development of other HTLV-1-associated diseases. Therefore, these patients should be followed up for a long time. ${ }^{1,4}$

It is recommended to perform the serology for HTLV-1 detection in patients with severe and resistant eczemas. Dermatologists should be familiar with these changes, since their recognition may lead to early diagnosis and adoption of important measures at the individual, family and collective levels. ${ }^{1,2}$ HTLV-I infection and associated diseases are remarkable for their severity and emerge as a serious public health problem.

\section{REFERENCES}

1. Bittencourt AL, Oliveira MF. Infective Dermatitis Associated to HTLV-I - A Review. An Bras Dermatol. 2001;76:723-732.

2. Araújo MG, Gonçalves DU, Carneiro-Proietti ABF, Proietti FA, Guedes ACM. Cutaneous manifestations in human T-cell lymphotropic vírus type 1 infection. An Bras Dermatol. 2008;83:393-407.

3. Carneiro-Proietti AB, Ribas JG, Catalan-Soares BC, Martins ML, Brito-Melo GE, Martins-Filho $\mathrm{OA}$, et al. Infecção e doença pelos vírus linfotrópicos humanos de células T (HTLV-1/II) no Brasil. Rev Soc Bras Med Trop. 2002;35:499-508.

4. Lee R, Schwartz RA. Human T-lymphotrophic virus type 1eassociated infective dermatitis: A comprehensive review. J Am Acad Dermatol. 2011;64:152-60.

5. Santos FLN, Lima FWM. Epidemiologia, fisiopatogenia e diagnóstico laboratorial da infecção pelo HTLV-I. J Bras Patol Med Lab. 2005;41:105-16.

6. Kendall EA, González E, Espinoza I, Tipismana M, Verdonck K, Clark D, et al. Early Neurologic Abnormalities Associated with Human T-Cell Lymphotropic Virus Type 1 Infection in a Cohort of Peruvian Children. J Pediatr. 2009;155:700-6.

7. Manns A, Hisada M, La Grenade L. Human T-lymphotropic virus type I infection. Lancet. 1999;353:1951-8.

8. de Oliveira Mde F, Vieira Md, Primo J, Siqueira IC, Carvalho EM, Farré L, et al. Flower cells in patients with infective dermatitis associated with HTLV-1. J Clin Virol. 2010;48:288-90.

9. Tschachler E. Human Retroviral Disease: Human T-Lymphotropic Viruses. In: Wolff K, Goldsmith LA, Katz SI, Gilchrest BA, Paller AS, Leffell, DJ. Fitzpatrick's Dermatology in General Medicine. New York: McGraw-Hill; 2008. p. 1923-7.

10. Goldman L, Ausiello D. Cecil: Tratado de Medicina Interna. 22 ed. Rio de Janeiro: Elsevier; 2005. P. 2336-41.

How to cite this article: Steglich RB, Tonoli RE, Souza PRM, Pinto GM, Riesgo RS. HTLV-1-associated infective dermatitis and probable HTLV-1-associated myelopathy in an adolescent female. An Bras Dermatol. 2015;90 (3 Suppl 1): S55-8. 